\title{
EDITORIAL
}

\section{Multidisciplinary studies and research needs}

Multi disciplinary or inter-disciplinary research requires a combination of methods and knowledge from more than one discipline. The main objective of publishing " International Journal of Multidiciplinary Studies" by the Fcaulty of Graduate Studies of the University of Sri Jayewardenepura is to encourage researchers working in different disciplines to focus their knowledge and research skills to work towards solving real world problems for which subject label cannot be attached. It is expected that this would immensely help the researchers to resolve important scientific problems through interactive efforts.

However to achieve this aim, initially the researchers have to be guided by a "road map" to select their research problems to address research needs of the country linked with the national development. Other than the "development activities" that enhance the economy of the country, there may be health related problems where immediate focus and emphasize has to be given by the scientists to find out causes and remedial measures. At present one such problem prevails in Sri Lanka; the Chronic Kidney Disease of unknown etiology (CKDu). It has become a serious health problem in the north central province of Sri Lanka with more than twenty thousand of affected people and a considerable number of deaths. It has created severe social and health problems in the area. However the actual cause for the issue is still unknown. In order to findout actual scientific reason for such problems it is essential to mobilize multidisciplinary research teams where chemists, geologists, hydrologists sociologists as well as medical professionals etc working interactively with similar groups in other countries where similar or allied problems prevail.

To engage in multidiciplinarty research, there are some important needs of the researchers such as easy access to data bases. This is a big problem mostly in the developing countries like Sri Lanka. Still we do not have a national data base from which the scientists could get required information fast. Government of Sri Lanka (GOSL) has to look into this issue in order to initiate such data bases allowing scientists to get required information using payment gateways. Even by paying a nominal fee there should be a way to get the required data such as meteorological data, digital map data, landuse data, etc through internet. In spite of the "high-tech" achievements of the country we are still far behind in this regard. GOSL could initiate development of a national data base as it will be extremely useful for researchers and all the development projects in progress. 
Researchers need high quality sensitive equipments to get acurate results in their experiments. At present availability of such equipment are limited only to some laboratories of the country. The trained scientists are distributed in different organizations of the country wasting their training \& experience that they received during their research trainings due to lack of access to required instruments to do their research. Some times even within the same institute or university some valuable equipment are underused without getting their maximum usage. Therefore to be in par with the development targets of the country, time has come to develop a crieteria to avoid wasting valuable resources without getting the maximum usage. Concept of central laboratory system could be adopted to overcome this undesirable development. However it is very important to provide trained technical staff and supporting staff also to maintan such equipment and also to assist the researchers. Separate funding sources are also needed for required consumables for such central laboratories for which GOSL could support.

Interactive research collaboration among different institutes is essential to solve problems of the country by getting the services of trained scientists from universities \& other organizations. It is very important to initiate such interactive research via consultancies to encourage required findings. It is always important to publish the research findings in the websites of the organizations to avoid repetition of the same work. Finally it should be emphasized that it is essential to provide necessary funding for research projects that contribute towards the development activities of the country through multidiciplinary research activities.

\section{Swarna Piyasiri}

Senior Professor \& Dean, Faculty of Graduate Studies, University of Sri Jayewardenepura 